\title{
Interaction Manifolds: Theory from Experiments
}

\author{
Cecily Morrison \\ Cambridge University \\ William Gates Building \\ $15 \mathrm{JJ}$ Thomson Avenue \\ Cambridge CB3 OFD UK \\ (+44) 1223763783 \\ Cecily.Morrison@cl.cam.ac.uk
}

\begin{abstract}
This poster builds on comparative ethnographic work of a multi-disciplinary medical team using a paper-based and a computer-based patient record system. It describes the design and preliminary results of an experiment aimed to help articulate an analytical construct that would describe the tradeoffs between a technological setup and a group's ability to negotiate an interaction among themselves.
\end{abstract}

\section{Categories and Subject Descriptors H.5.3 [Group and Organization Interfaces]}

\section{General Terms}

Performance, Design, Human Factors, Theory

\section{Keywords}

CSCW, Interaction Manifold

\section{INTRODUCTION}

In a previous ethnographic study [1], we examined how a multidisciplinary medical team negotiated interaction amongst themselves while using a patient medical record to decide on future treatment. We compared how the physical interactions, (or non-verbal behaviours), changed when a paper-based system was used as opposed to a computer-based one. The result of the work suggested a need for an analytical construct that identifies the trade-offs between the configuration of a technological setup and the abilities of a group to negotiate interaction. As a first step in developing this construct, the interaction manifold, we are preparing a number of experiments. Using a motion capture system that enables us to track the position of participants' entire bodies in 3-dimensional space that is linked with video, we are able to evaluate a group's physical interactions in a given circumstance both qualitatively and quantitatively. After summarizing the research questions posed by our previous study, we will describe the experiment and preliminary results.

\author{
Alan F. Blackwell \\ Cambridge University \\ William Gates Building \\ $15 \mathrm{JJ}$ Thomson Avenue \\ Cambridge CB3 OFD UK \\ (+44) 1223763783 \\ Alan.Blackwell@cl.cam.ac.uk
}

\section{BACKGROUND}

The ethnographic study that this work builds on compared interaction around a paper-based patient medical record system to a computer-based one in an intensive care unit. We specifically looked at physical interactions - that is: group formation, upper-body orientation, gesture manipulation, object manipulation and posture. Analysis of the paper-based system usage demonstrated the importance of physical interactions in seamlessly negotiating conversation. It also showed that Kendon's F-formation [2], a framework describing non-verbal behaviour in group interaction, fits well. However, this was not the case with the computer-based system where the group was forced to split due to the position of the display, as shown in figure 1 , causing a break down in communication.

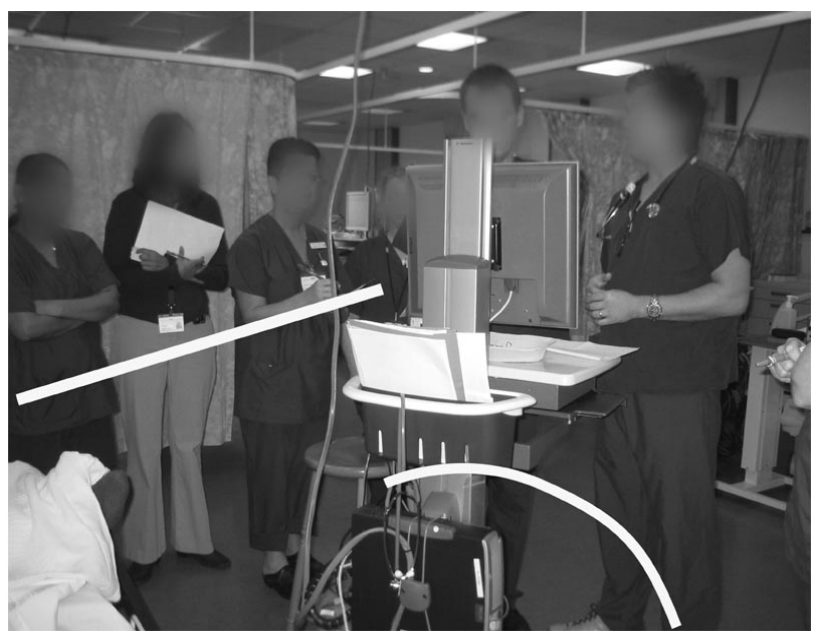

Figure 1: Group interaction around a computer-based medical record system

The split removed physical interactions as a viable means of communication, blocking members from participating in the interaction. The inability of group members to monitor each other's physical (non-verbal) interactions caused a reduction in parallel work and less integration with those switching between reviewing, taking notes and the group conversation. The solution to static display devices is generally mobile devices which allow people to configure themselves as need be. However, it is not clear from our ethnographic research whether this will solve the problem. We conjecture that -- looking at different content, no central source of orientation, and an inability to monitor what information that others are using -will decrease group cohesion and thus, the effectiveness of the interaction. The experiment described below aims to understand more precisely whether mobile handheld devices, 
such as Emanotech's new device MedTab [3], can solve the problems articulated above. We would therefore like to test how physical interactions differ when a group of 3 performs a cooperative task using a large, wall-projected display versus having a shared screen displayed on a personal handheld device that each participant holds individually.

\section{EXPERIMENTAL DESIGN}

\subsection{Setup}

Each participant wears a hat, one glove, a belt, a shoulder pad, and shoe covers fitted with reflective dots whose 3-dimensional coordinates can be tracked by a Vicon motion capture system within a 3 × 3 meter area, illustrated below in figure 2 .

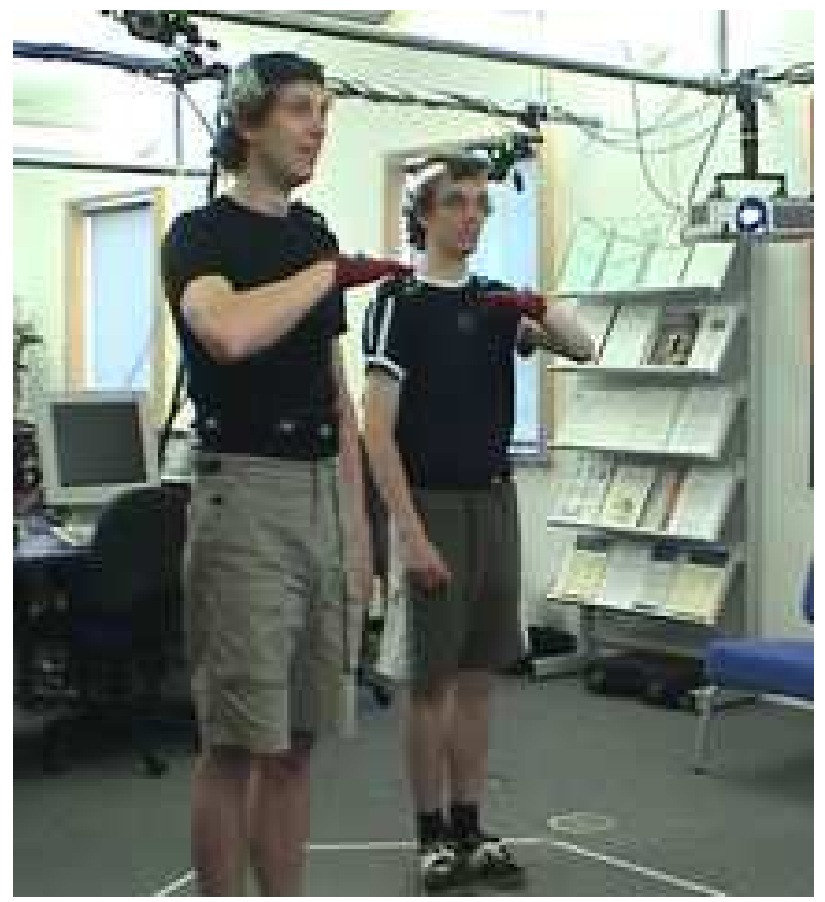

Figure 2: Experimental Setup

The large display is projected $50 \mathrm{~cm}$ in front of the motion capture space. The handheld displays are standard PDAs running VNC. In both cases, the display will be created through a java program running on the main computer and projected or sent as appropriate.

\subsection{Task}

Participants cooperatively control one on-screen pen with their body movements in a drawing program. Each participant can manipulate either the $\mathrm{x}$-component, $\mathrm{y}$-component, or speed of the direction vector by changing the angle of their hand to their hip and the colour, width, or alpha value by moving in space.
They are asked to do the following two exercises: (1) draw their dream house; (2) draw an animal that is a cross between their three favourite animals. The order of exercises and display types are randomised.

\section{EVALUATION}

Participants will be videoed and log files kept of their head, torso, and foot movements as well as their absolute position in space. Using this data, we will analyse the following:

(1) changes in group formation by visualizing each participant's position and orientation;

(2) completion times;

(3) number of times and degree that participants turn their heads, upper torso, or whole body (feet) towards each other;

(4) conversation analysis.

Visualisations will be rendered to examine each of the above categories individually as well as, all data will be fed into Replayer, an application that allows simultaneous viewing of different media at any given timestamp.

\section{PRELIMINARY RESULTS}

As of the writing of this paper, a preliminary experiment has been completed to test the usability of the drawing program and provide initial feedback from the participants. 2 pairs of students used the program with the wall projection. Both pairs found the program very enticing and after 1.5 hours we had to request that they finish. The students, even the nonmathematical ones, had no problem controlling the system, taking about 10 minutes to adjust. We saw very different styles of cooperation and therefore different postures, body orientations and speech styles. It became clear that cooperation styles will need to be accounted for in the analysis of the full experiment.

\section{ACKNOWLEDGMENTS}

We are grateful for the support of Papworth Critical Care Unit, Papworth Anaesthetic Research, and head consultant Alain Vuylsteke, who initiated this project. This research is sponsored by Boeing Corporation and IMDsoft, and is being conducted in collaboration with David Good and Alice McGowan (Social and Political Science) and Matthew Jones (Judge Business School).

\section{REFERENCES}

[1] Morrison, C. Blackwell, A. F. (2007). Manifolds of Social Interaction in Physical-Digital Environments. (work-inprogress)

[2] Kendon, A. (1990). Conduction Interaction: Patterns of Behavior in focused encounters, Cambridge University Press, Cambridge, UK.

[3] Emano Tec Inc. (2007). www.emanotec.com/medtab.htm 